\title{
Hepatic stiffness in the bidirectional cavopulmonary circulation: The Liver Adult-Pediatric-Congenital-Heart-Disease Dysfunction Study group
}

\author{
Shaija S. Kutty, MD, ${ }^{a}$ Ming Zhang, MD, PhD, ${ }^{b}$ David A. Danford, MD, ${ }^{b}$ Rimsha Hasan, MD, ${ }^{b}$ \\ Kim F. Duncan, MD, ${ }^{\mathrm{c}}$ John D. Kugler, MD, ${ }^{\mathrm{b}}$ Ruben E. Quiros-Tejeira, MD, ${ }^{\mathrm{a}}$ and Shelby Kutty, MD
}

\section{ABSTRACT}

Objectives: We hypothesized that hepatic injury in single-ventricle CHD has origins that predate the Fontan operation. We aimed to measure hepatic stiffness using ultrasound and shear wave elastography (SWE) in a bidirectional cavopulmonary connection (BCPC) cohort.

Methods: Subjects were prospectively recruited for real-time, hepatic, ultrasound-SWE for hepatic stiffness $(\mathrm{kPa})$ and echocardiography. Doppler velocities, a velocity-time integral, flow volume, and resistive index, pulsatility index, and acceleration index were measured in celiac and superior mesenteric arteries, and in the main portal vein (MPV). Comparisons were made among subjects who had BCPC, subjects who were healthy, and a cohort of patients who had undergone the Fontan procedure.

Results: Forty subjects (20 patients who had BCPC; 20 age- and gender-matched control subjects) were studied. The hepatic stiffness in BCPC was elevated, compared with that in control subjects (7.2 vs $5.7 \mathrm{kPa} ; P=.039)$. Patients who had BCPC had significantly higher celiac artery resistive index (0.9 vs 0.8 ; $P=.002)$; pulsatility index (2.2 vs $1.7 ; P=.002)$; and systolic-diastolic flow ratio (10.1 vs $5.9 ; P=.002$ ), whereas the superior mesenteric artery acceleration index (796 vs $1419 \mathrm{~mL} / \mathrm{min}$ in control subjects; $P=.04$ ) was lower. An elevated resistive index $(0.42$ vs $0.29 ; P=.002)$ and pulsatility index $(0.55$ vs $0.35 ; P=.001)$ were seen in MPV, whereas MPV flow was reduced $(137.3 \mathrm{vs} 215.7 \mathrm{~mL} / \mathrm{min}$ in control subjects; $P=.036$ ). A significant correlation was found for hepatic stiffness with right atrial pressure obtained at catheterization $(P=.002)$. Comparison with patients who underwent the Fontan procedure showed patients who had BCPC had lower hepatic stiffness $(7.2$ vs $15.6 \mathrm{kPa} ; P<.001)$.

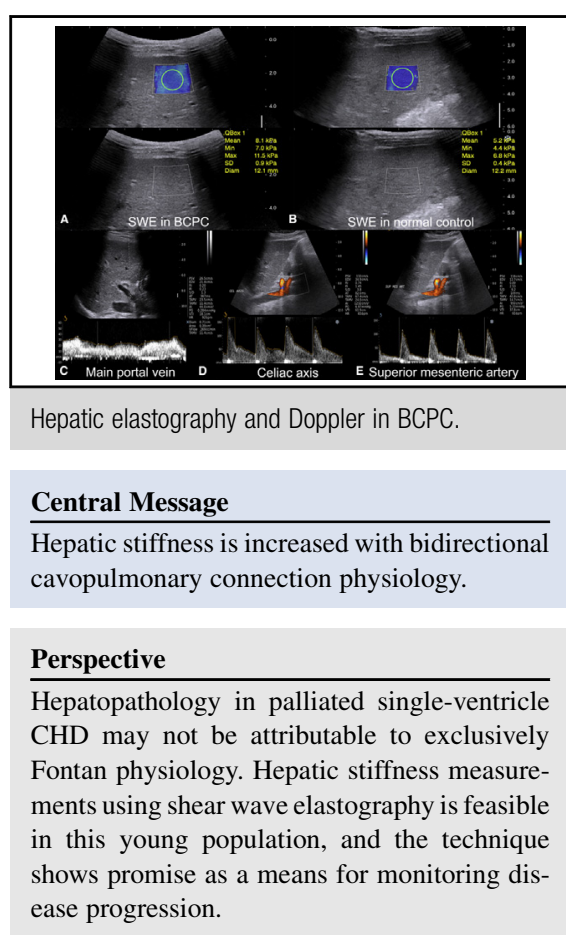

See Editorial Commentary page 685.

See Editorial page 621.

Conclusions: Hepatic stiffness is increased with BCPC physiology, and this finding raises concerns that hepatopathology in palliated, single-ventricle CHD is not exclusively attributable to Fontan physiology. Hepatic stiffness measurements using SWE are feasible in this young population, and the technique shows promise as a means for monitoring disease progression. (J Thorac Cardiovasc Surg 2016;151:678-84)

The bidirectional cavopulmonary connection (BCPC) is a palliative procedure for single-ventricle congenital heart disease (CHD), typically performed on patients aged 3 to

From the Departments of ${ }^{\mathrm{a}}$ Pediatric Gastroenterology, ${ }^{\mathrm{b}}$ Pediatric Cardiology, and ${ }^{\mathrm{c} C a r d i o v a s c u l a r}$ Surgery, University of Nebraska College of Medicine and Children's Hospital and Medical Center, Omaha, Neb.

This study was funded in part by the Children's Hospital and Medical Center Foundation Grant, Omaha, Neb. S.K. receives support from the American Heart Association. Supersonic Imagine, Inc, generously provided equipment and technical support.
6 months, to provide adequate prograde pulmonary blood flow until Fontan palliation is accomplished 3 to 4 years later. The procedure consists of an end-to-side connection

Received for publication June 12, 2015; revisions received Aug 23, 2015; accepted for publication Sept 12, 2015; available ahead of print Oct 26, 2015.

Address for reprints: Shelby Kutty, MD, University of Nebraska Medical Center, and Children's Hospital and Medical Center, 8200 Dodge St, Omaha, NE 68114 (E-mail: skutty@unmc.edu). $0022-5223 / \$ 36.00$

Copyright (C) 2016 by The American Association for Thoracic Surgery http://dx.doi.org/10.1016/j.jtcvs.2015.09.079 

Abbreviations and Acronyms
$\mathrm{BCPC}=$ bidirectional cavopulmonary connection
$\mathrm{CHD}=$ congenital heart disease
MPV = main portal vein
SWE = shear wave elastography

between the superior vena cava and the undivided right pulmonary artery, and flow from the heart into the pulmonary arteries is interrupted in most patients.

Unfortunately, hepatic abnormalities after the Fontan operation are common ${ }^{1-5}$; these include markedly higher risk for nonalcoholic cirrhosis. ${ }^{6}$ Progressive hepatic failure and even hepatocellular carcinoma has occurred in these young patients. ${ }^{1,5,7,8}$ The pathologic changes in the liver after a Fontan operation consist of sinusoidal dilatation and fibrosis. These changes are presumably related to high venous pressures from high resistance to hepatic venous drainage, also called "hepatic afterload," but hypoxemia and diminished cardiac output may be important factors in pathogenesis. ${ }^{2,9,10}$

The clinical importance of liver disease in singleventricle $\mathrm{CHD}$ is recognized increasingly. However, very little is known about the origin and progression of liver fibrosis in this population. Hepatic failure in singleventricle heart disease patients influences surgical choices, including Fontan revision, heart transplantation, and heartliver transplantation, so noninvasive means for evaluating the liver in single-ventricle CHD are of great interest.

The Liver Adult-Pediatric-Congenital-Heart-DiseaseDysfunction Study (LADS) group at our institution recently reported the feasibility of shear wave elastography (SWE) for noninvasive assessment of hepatic stiffness. ${ }^{10}$ Analysis of SWE and ultrasound and duplex flow dynamics suggests that Fontan physiology produces sufficient elevation of hepatic afterload to result in changes in hepatic stiffness and histology. ${ }^{10}$ After the Fontan procedure, hepatic stiffness is markedly increased; flow volume in the main portal vein is decreased; and celiac and mesenteric arterial resistive indices are higher. ${ }^{10}$ Associations of elevated hepatic stiffness with unfavorable Fontan hemodynamics and advanced liver fibrosis are well documented. ${ }^{11,12}$ Hepatic stiffness in a single ventricle at the BCPC palliation stage has not been reported, but may provide insights into the pathogenesis of this process.

We hypothesized that hepatic stiffness in a palliated single ventricle, as measured by ultrasound elastography, is elevated before the Fontan operation. The purpose of this study was to use SWE prospectively, to measure hepatic stiffness in infants and children who have BCPC physiology, for comparison with age- and gender-matched healthy control subjects, and with a historical cohort of patients who underwent the Fontan procedure, from our institution. ${ }^{10}$ In addition, we sought to correlate hepatic stiffness and vascular Doppler indices with patient age, time from $\mathrm{BCPC}$, and pre-BCPC cardiac catheterization hemodynamic findings.

\section{METHODS \\ Study Population}

This was a prospective, single-center research study, approved by an institutional review board, conducted between May 2013 and September 2014. Patients with BCPC physiology were prospectively enrolled for research liver ultrasound-SWE imaging at the time of routine outpatient clinical follow-up. Age- and gender-matched healthy volunteer infants and children (serving as a control group) were recruited in response to an advertisement asking for participation, which was placed in the institution's (of the review board) employee newsletter. Informed, written consent was obtained from the parents or legal guardians of all recruited patients and healthy subjects. Complete transthoracic echocardiograms were performed in the BCPC subjects within 3 months of liver ultrasound-SWE, using a GE Vivid E9 Ultrasound System (GE Healthcare, Milwaukee, Wis).

\section{Hepatic Ultrasound and SWE Protocol}

A comprehensive liver ultrasound examination with duplex, followed by SWE, was performed using the ultrasound-SWE system (Aixplorer, SuperSonic Imagine, Bothell, Wash), per our protocol. ${ }^{10}$ Real-time B-mode imaging, with Doppler assessments of the celiac axis, the superior mesenteric artery, and the main portal vein (MPV) was performed. A broad-bandwidth curved transducer (SC6-1, SuperSonic Imagine) was used for generation of transient shear waves with simultaneous real-time B-mode imaging.

Quantitative viscoelasticity mapping of the liver was performed from the shear wave propagation, generating a real-time anatomic reference gray-scale image and an elastogram color map. At least 3 SWE image acquisitions were made in each study. A-single, experienced sonographer (one of the investigators) performed all acquisitions. The study was transferred to a Macintosh computer (Macbook Pro, Apple, Inc, Cupertino, Calif), for SWE quantification using the OsiriX DICOM (Digital Imaging and Communications in Medicine) review software and Q-box plug-in (OsiriX, Pixmeo Sàrl, Geneva, Switzerland). The Q-box was positioned over an area of relative homogeneous elastogram, within a zone of uniform liver parenchyma. The SWE gain (70\%), Q-box diameter (12 mm), elastogram range $(0-40 \mathrm{kPa})$, and depth $(4-7 \mathrm{~cm})$ were set to the default setting, per manufacturer recommendations. The mean elastogram value was measured, expressed in terms of Young's modulus $(\mathrm{kPa})$ in the area delineated by the Q-box. The measurement was repeated 3 times, to obtain 3 independent SWE maps of the patient in the same scanning plane, and the average of the 3 independent mean elastogram values was taken as the final hepatic stiffness for each patient.

\section{Statistical Analysis}

Data are expressed as mean \pm SDs with ranges. The nonpaired Student $t$ test was used for comparison of patients who had BCPC with the control group and the group who underwent the Fontan procedure. Univariate least-square linear regressions of hepatic stiffness and vascular Doppler indices against patient age, body surface area, and duration of BCPC were performed. Statistical analysis was performed using Minitab 16.1 (Minitab, Inc, State College, Pa).

\section{RESULTS}

In all, 40 subjects were studied, including 20 patients who had BCPC physiology, and 20 age- and gender-matched 
TABLE 1. Subject demographics

\begin{tabular}{lccr}
\hline \multicolumn{1}{c}{ Variable } & Control group & Patients & $\begin{array}{c}\boldsymbol{P} \\
\text { value }\end{array}$ \\
\hline Age $(\mathrm{mo})$ & $24 \pm 17(5-53)$ & $24 \pm 16(6-52)$ & .352 \\
Height $(\mathrm{cm})$ & $84.7 \pm 17.4(61-109)$ & $81.8 \pm 15.1(59-107)$ & .567 \\
Weight $(\mathrm{kg})$ & $12.2 \pm 3.8(6.1-17.7)$ & $11.3 \pm 3.8(5.3-18.1)$ & .451 \\
Body surface & $0.54 \pm 0.13(0.33-0.73)$ & $0.49 \pm 0.14(0.28-0.73)$ & .321 \\
$\quad$ area $\left(\mathrm{m}^{2}\right)$ & & & \\
\hline Values are mean $\pm \mathrm{SD}$ (range), unless otherwise indicated. Total subjects $=40$.
\end{tabular}

controls. A total of 22 patients were in the institution awaiting Fontan completion during the 18-month period of this investigation. Demographics for all subjects are summarized in Table 1. The average time since BCPC for patients was 18 months (range: 3-49 months). Echocardiography (mean interval: 0.5 months from the liver ultrasoundSWE test) revealed normal ventricular systolic function in $18(90 \%)$ patients, and mildly depressed function in 2 $(10 \%)$.

The primary cardiac diagnoses in patients were as follows: hypoplastic left heart syndrome, or its variants $(\mathrm{n}=10)$; pulmonary atresia with intact ventricular septum
( $\mathrm{n}=4)$; tricuspid atresia $(\mathrm{n}=4)$; and double-inlet left ventricle $(n=2)$. The ventricular morphology was left ventricular in $10(50 \%)$ and right ventricular in 10 $(50 \%)$. Atrioventricular valve regurgitation was mild or less than mild in $18(90 \%)$ patients, and moderate in 2 $(10 \%)$. All except 1 patient $(n=19)$ had pre-BCPC cardiac catheterization as part of their clinical care.

Measurements using SWE were obtained for all subjects. The hepatic stiffness in patients who had BCPC was elevated, compared with that of healthy subjects: $7.2 \pm 2.5 \mathrm{kPa}$ (range: $4.7-14.9 \mathrm{kPa}$ ) versus $5.7 \pm 0.7 \mathrm{kPa}$ (range: 4.2-7.2 kPa), respectively $(P=.039$ ) (Figure 1 ). Comparison of hepatic stiffness with a historical cohort of subjects who had undergone the Fontan procedure ${ }^{10}$ showed that patients who had BCPC had lower hepatic stiffness (mean: 7.2 vs $15.6 \mathrm{kPa}$, respectively; $P<.001$ ). The historical cohort consisted of 41 patients (29 male; $12 \mathrm{fe}$ male; age $13.8 \pm 6.3$ years; time interval of ultrasound and SWE from Fontan: $11 \pm 6$ years). The Fontan types were extracardiac in 19 patients $(46 \%)$, and lateral tunnel in $22(54 \%)$; the ventricular morphology was left ventricular in $25(61 \%)$, and right ventricular in $16(39 \%)$. Ten

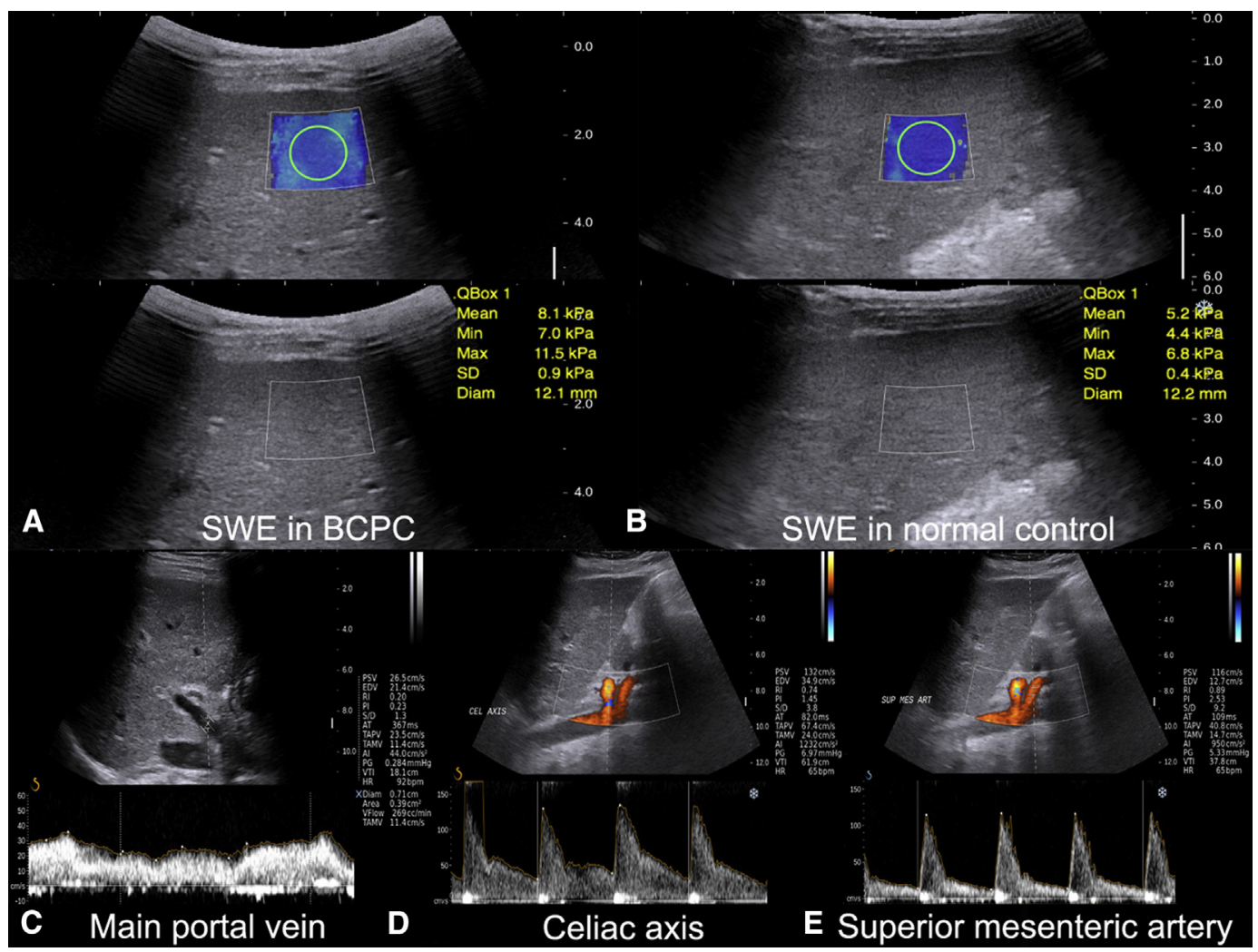

FIGURE 1. Shear wave elastogram and vascular Doppler indices are shown. Elastogram maps show hepatic stiffness measurements by SWE in (A) a subject who had BCPC, and (B) a healthy subject. Hepatic ultrasound Doppler measurements are shown for (C) the main portal vein; (D) the celiac artery; and (E) the superior mesenteric artery. Min, Minimum; Max, maximum; Diam, diameter; SWE, shear wave elastography; $B C P C$, bidirectional cavopulmonary connection; $P S V$, peak systolic velocity; $E D V$, end-diastolic velocity; $R I$, resistive index; $P I$, pulsatility index; $A T$, acceleration time; $T A P V$, time average peak velocity; TAMV, time average mean velocity; AI, acceleration index; PG, peak gradient; VTI, velocity-time integral; HR, heart rate; VFlow, flow volume. 
patients had an open fenestration in the Fontan baffle. Atrioventricular regurgitation was none or mild in $36(88 \%)$ patients, and moderate in $5(12 \%)$ patients.

Vascular Doppler indices in patients who had BCPC, compared with the control group show that they had a significantly higher celiac artery resistive index (0.87 vs $0.80 ; \quad P=.002)$; pulsatility index (2.21 vs 1.76 ; $P=.002)$, and systolic-diastolic flow ratio (10.1 vs 5.9; $P=.002)$. In the superior mesenteric arteries, the acceleration index in patients was significantly lower than that in control subjects (796 $\pm 441 \mathrm{vs} 1419 \pm 671 \mathrm{~cm} / \mathrm{s}^{2}$, respectively; $P=.02$ ), suggesting decreased superior mesenteric artery flow. In patients who had BCPC, compared with healthy subjects, the MPV resistive index $(0.42$ vs 0.29 ; $P=.002)$ and pulsatility index $(0.55$ vs $0.35 ; P=.001)$ were elevated, whereas the MPV flow volume was decreased (137.3 vs $215.7 \mathrm{~mL} /$ minute; $P=.036)$, as well as the velocity-time integral $(17.1 \pm 3.5$ vs $21.3 \pm 4.8$; $P=.008)$. The MPV diameter and flow were larger in patients who had BCPC with right-ventricular morphology ( 0.54 vs $0.44 \mathrm{~cm} ; P=.02$ ), compared with those who had left-ventricular morphology (174.2 vs $75.7 ; P=.040)$ (Table 2).

Evaluation of the relationship of hepatic stiffness and duplex indices with age and body surface area in patients and healthy subjects shows that the MPV diameter and flow were positively correlated with patient age $(P<.001$ and $P=.04$, respectively), duration of palliation $(P=.005)$, and body surface area $(P=.04)$. Hepatic stiffness was correlated with MPV resistive and pulsatility index $(P=.03)$ (Table 3). Evaluation of the association of hepatic stiffness with pre-BCPC cardiac catheterization showed a significant correlation of SWE with right atrial pressure $(P=.002$; Table 4$)$. Positive correlations were found, of MPV diameter, MPV flow, and celiac artery velocity-time integral with body surface area, and of MPV resistive index, pulsatility index, and right atrial pressure with hepatic stiffness (Figure 2). No significant change was seen in hepatic stiffness or other duplex indices with differences in either duration of BCPC or ventricular morphology.

\section{DISCUSSION}

Determination of hepatic stiffness, using SWE, was feasible and revealing for children who had singleventricle $\mathrm{CHD}$ at the $\mathrm{BCPC}$ stage. Patients who have BCPC have mildly elevated hepatic stiffness, compared with healthy subjects. Single ventricles often exhibit systolic and diastolic dysfunction, and they can be burdened with volume loading from regurgitant cardiac valves, and with pressure loading from distal arterial stenoses. Cardiac output may be thus impaired, and compensatory changes in preload may result. Even before BCPC occurs, right atrial pressure may be elevated. We observed that hepatic
TABLE 2. Vascular Doppler indices in the celiac artery, superior mesenteric artery, and main portal vein in all subjects

\begin{tabular}{cccc}
\hline & & & $\boldsymbol{P}$ \\
Variable & Control group & Patients & value \\
\hline CA-RI & $0.80 \pm 0.07(0.71-0.90)$ & $0.87 \pm 0.05(0.77-0.94)$ & $.002^{*}$ \\
CA-PI & $1.76 \pm 0.37(1.27-2.39)$ & $2.21 \pm 0.34(1.85-2.88)$ & $.002^{*}$ \\
CA-SDVR & $5.9 \pm 2.2(3.4-10.2)$ & $10.1 \pm 3.7(4.4-16.5)$ & $.002^{*}$ \\
CA-AI & $1167 \pm 448(338-1811)$ & $1020 \pm 881(355-2665)$ & .709 \\
$\quad\left(\mathrm{~cm} / \mathrm{s}^{2}\right)$ & & & \\
CA-VTI & $27.2 \pm 12.7(10.2-60.0)$ & $24.6 \pm 9.5(12.6-50.3)$ & .513 \\
$\quad(\mathrm{~cm})$ & & & \\
SMA-RI & $0.87 \pm 0.05(0.74-0.99)$ & $0.87 \pm 0.07(0.73-0.95)$ & .862 \\
SMA-PI & $2.25 \pm 0.44(1.44-3.30)$ & $2.43 \pm 0.54(1.57-3.44)$ & .424 \\
SMA-SDVR & $8.5 \pm 3.4(3.8-19.1)$ & $10.5 \pm 6.8(3.8-26.7)$ & .440 \\
SMA-AI & $1419 \pm 671(616-3236)$ & $796 \pm 441(354-1602)$ & $.015^{*}$ \\
$\quad\left(\mathrm{~cm} / \mathrm{s}^{2}\right)$ & & & \\
SMA-VTI & $26.3 \pm 7.3(10.8-41.6)$ & $20.8 \pm 7.0(15.1-34.4)$ & .131 \\
$(\mathrm{~cm})$ & & & \\
MPV-RI & $0.29 \pm 0.10(0.13-0.55)$ & $0.42 \pm 0.13(0.25-0.68)$ & $.002^{*}$ \\
MPV-PI & $0.35 \pm 0.15(0.14-0.83)$ & $0.55 \pm 0.18(0.29-0.83)$ & $.001^{*}$ \\
MPV-SDVR & $1.5 \pm 0.4(1.2-2.9)$ & $2.1 \pm 1.3(1.3-6.3)$ & .069 \\
MPV-AI & $49.9 \pm 39.5(10.2-109.0)$ & $44.6 \pm 16.0(23.7-73.2)$ & .599 \\
$\left(\mathrm{~cm} / \mathrm{s}^{2}\right)$ & & & \\
MPV-VTI & $21.3 \pm 4.8(13.1-29.4)$ & $17.1 \pm 3.5(13.2-24.7)$ & $.008^{*}$ \\
$(\mathrm{~cm})$ & & & \\
MPV- & $0.54 \pm 0.10(0.39-0.81)$ & $0.49 \pm 0.09(0.36-0.72)$ & .115 \\
diameter & & & \\
$(\mathrm{cm})$ & & & \\
MPV-flow & $215.7 \pm 91.4(95.9-552.0)$ & $137.3 \pm 77.0(62.2-285.0)$ & $.036^{*}$ \\
$(\mathrm{~mL} / \mathrm{min})$ & & & \\
\hline Vas & & & \\
\hline
\end{tabular}

Values are mean \pm SD (range), unless otherwise indicated. $C A$, Celiac artery; $R I$, resistive index; $P I$, pulsatility index; $S D V R$, systolic-to-diastolic velocity ratio; $A I$, acceleration index; $V T I$, velocity-time integral; $S M A$, superior mesenteric artery; $M P V$, main portal vein. $* P<.05$.

stiffness was correlated with elevated pre-BCPC right atrial pressure. To the extent that single-ventricle CHD at the BCPC stage might lead to hepatic afterload elevation, associated changes may occur in hepatic stiffness. Such changes do not seem to be nearly as large as those that occur after the Fontan operation, but even at the time of BCPC, a multifactorial hepatic burden may be present, with contributions from prior clinical illnesses and episodes of cardiopulmonary bypass, vascular congestion, and chronic hypoxemia.

This investigation did not determine hepatic stiffness in patients before they had BCPC, so BCPC itself cannot be inferred to be a cause of hepatic stiffness. In fact, evidence indicates that the hepatic changes are a result of chronically elevated systemic venous pressure, independent of BCPC. The correlation of stiffness with the right atrial pressure at the point of pre-BCPC catheterization suggests that hepatic congestion may predispose a patient to liver injury in a single-ventricle well before the Fontan operation has been completed. In addition, we identified other markers of hepatic congestion in patients who had BCPC, including 
TABLE 3. Relationship of hepatic stiffness and vascular Doppler indices with age and BSA

\begin{tabular}{|c|c|c|c|c|c|c|}
\hline \multirow[b]{2}{*}{ Measure } & \multicolumn{2}{|c|}{$\begin{array}{c}\text { Age at } \\
\text { study (mo) }\end{array}$} & \multicolumn{2}{|c|}{ BSA } & \multicolumn{2}{|c|}{$\begin{array}{c}\text { SWE } \\
\text { value }(\mathrm{kPa})\end{array}$} \\
\hline & $\begin{array}{c}\text { Adj } \\
r^{2}(\%)\end{array}$ & $\begin{array}{c}P \\
\text { value }\end{array}$ & $\begin{array}{c}\text { Adj } \\
r^{2}(\%)\end{array}$ & $\begin{array}{c}P \\
\text { value }\end{array}$ & $\begin{array}{c}\text { Adj } \\
r^{2}(\%)\end{array}$ & $\begin{array}{c}P \\
\text { value }\end{array}$ \\
\hline SWE value $(\mathrm{kPa})$ & 0 & .885 & 0 & .948 & - & - \\
\hline CA-RI & 1.0 & .258 & 3.0 & .168 & 0 & .386 \\
\hline CA-PI & 0 & .795 & 0 & .592 & 0 & .839 \\
\hline CA-SDVR & 0 & .839 & 0 & .591 & 0 & .511 \\
\hline CA-AI $\left(\mathrm{cm} / \mathrm{s}^{2}\right)$ & 1.9 & .212 & 0 & .433 & 1.6 & .228 \\
\hline CA-VTI (cm) & 8.0 & .06 & 13.6 & $.02 *$ & 2.9 & .171 \\
\hline SMA-RI & 0 & .936 & 0 & .976 & 0 & .939 \\
\hline SMA-PI & 0 & .466 & 0 & .418 & 5.7 & .111 \\
\hline SMA-SDVR & 1.5 & .242 & 0 & .338 & 0 & .778 \\
\hline SMA-AI $\left(\mathrm{cm} / \mathrm{s}^{2}\right)$ & 15.2 & $.021 *$ & 9.1 & .061 & 0 & .485 \\
\hline SMA-VTI (cm) & 0 & .615 & 1.9 & .226 & 0 & .618 \\
\hline MPV-RI & 5.7 & .077 & 2.0 & .190 & 8.6 & $.039 *$ \\
\hline MPV-PI & 3.0 & .147 & 0 & .336 & 9.6 & $.031 *$ \\
\hline MPV-SDVR & 4.9 & .093 & 2.6 & .163 & 0 & .714 \\
\hline MPV-AI $\left(\mathrm{cm} / \mathrm{s}^{2}\right)$ & 5.8 & .076 & 9.4 & $.032 *$ & 0 & .785 \\
\hline MPV-VTI (cm) & 7.0 & .05 & 4.9 & .093 & 0.7 & .264 \\
\hline $\begin{array}{l}\text { MPV-diameter } \\
\quad(\mathrm{cm})\end{array}$ & 27.1 & $<.001^{*}$ & 27.5 & $<.001^{*}$ & 0 & .613 \\
\hline $\begin{array}{l}\text { MPV-flow } \\
\quad(\mathrm{mL} / \mathrm{min})\end{array}$ & 8.2 & $.043^{*}$ & 8.5 & $.040 *$ & 0 & .324 \\
\hline
\end{tabular}

$B S A$, Body surface area; $S W E$, shear wave elastography; $A d j$, adjusted; $C A$, celiac artery; $R I$, resistive index; $P I$, pulsatility index; $S D V R$, systolic-diastolic velocity ratio; $A I$, acceleration index; VTI, velocity-time integral; SMA, superior mesenteric artery; $M P V$, main portal vein. $* P<.05$.

increased MPV diameter and reduced MPV flow. These early physiologic derangements in palliated singleventricle CHD persist or increase after the Fontan operation. $^{10}$

Liver histopathology in patients who undergo the Fontan operation has demonstrated chronic passive congestion, centrilobular necrosis, and cirrhosis, and the severity of changes was correlated with right atrial pressures. ${ }^{7}$ High

TABLE 4. Association of hepatic stiffness with cardiac catheterization indices in the group who had bidirectional cavopulmonary connection

\begin{tabular}{|c|c|c|c|c|}
\hline \multirow[b]{2}{*}{ Parameter } & \multirow[b]{2}{*}{ Mean \pm SD } & \multirow[b]{2}{*}{ Range } & \multicolumn{2}{|c|}{$\begin{array}{l}\text { Regression with } \\
\text { hepatic stiffness }\end{array}$} \\
\hline & & & $\boldsymbol{r}$ & $P$ value \\
\hline Right atrial pressure $(\mathrm{mm} \mathrm{Hg})$ & $6.8 \pm 1.6$ & $5-11$ & 0.715 & $.002 *$ \\
\hline $\begin{array}{l}\text { Ventricular end-diastolic } \\
\text { pressure }(\mathrm{mm} \mathrm{Hg})\end{array}$ & $7.9 \pm 1.9$ & $6-12$ & 0.038 & .882 \\
\hline Mixed venous saturation $(\%)$ & $49.7 \pm 6.0$ & $40-59$ & -0.420 & .083 \\
\hline Systemic saturation $(\%)$ & $74.3 \pm 5.0$ & $65-86$ & -0.127 & .603 \\
\hline $\begin{array}{l}\text { Pulmonary vascular } \\
\text { resistance (Wood units.m²) }\end{array}$ & $1.9 \pm 0.6$ & $1.0-2.7$ & -0.401 & .099 \\
\hline Qp indexed $\left(\mathrm{L} / \mathrm{min} / \mathrm{m}^{2}\right)$ & $2.4 \pm 0.9$ & $1.6-4.0$ & -0.230 & .343 \\
\hline Qs indexed $\left(\mathrm{L} / \mathrm{min} / \mathrm{m}^{2}\right)$ & $2.9 \pm 0.5$ & $2.1-4.3$ & -0.338 & .170 \\
\hline Qp:Qs & $0.8 \pm 0.5$ & $0.6-1.2$ & -0.017 & .947 \\
\hline
\end{tabular}

$S D$, Standard deviation; $Q p$, pulmonary blood flow; $Q s$, systemic blood flow. $* P<.05$. venous pressure likely plays a major role in the progression of hepatic injury in single-ventricle CHD, because cirrhosis is more common in patients who have high venous pressure, and the severity of fibrosis is correlated with hepatic venous pressures. ${ }^{7,13}$ A recent observation indicates that a substantial percentage of patients who died within 1 month after undergoing the Fontan operation had significant hepatic fibrosis, suggesting that fibrosis in those patients resulted from pre-Fontan operation insults. ${ }^{14}$ Markers of pre-Fontan operation morbidity, including length of hospitalization after heart surgery, and right atrial pressure, were significantly associated with the degree of portal fibrosis in the patients who died. ${ }^{14}$

Although hepatic stiffness in patients who have BCPC is higher than it is in healthy children, it does not approach the levels documented after patients have undergone the Fontan operation. Clinical management implications of this observation are debatable, but if exposure of the liver to the more-adverse Fontan physiology could be delayed, perhaps this would slow the progression of liver disease. When functional status is satisfactory, the best approach may be to remain with BCPC indefinitely, rather than to perform the Fontan operation simply because the patient has reached an age and size at which technical success is likely. An appropriate investigation would be to evaluate the feasibility of hepatic-exclusion Fontan techniques, which might channel the infrahepatic inferior vena cava blood to the lungs, while retaining the liver's venous drainage to the heart. If needed to prevent pulmonary arteriovenous fistula formation, hepatic factor could be supplied to the lungs with a small systemic-to-pulmonaryartery shunt, or even by creating an upper-extremity arteriovenous fistula.

The relatively small sample size was a limitation of this study. Nevertheless, due to an extraordinarily high recruitment rate, the sample constitutes $>90 \%$ of all patients who had BCPC at our institution during the period of investigation. The findings are therefore expected to be truly representative of those of an unselected clinical cohort of such patients. No histopathologic comparisons validated hepatic stiffness measurements in BCPC. Performing liver biopsy in these infants and children was not feasible, for ethical reasons, their small size, and risks associated with the anesthesia requirements.

This study does not demonstrate that hepatic stiffness is either associated with worse clinical outcomes, or is of clinical benefit as a surrogate marker for liver injury, in this population. Hepatic stiffness without fibrosis could result from portal hypertension, or from nonfibrotic inflammation. However, histology data in patients undergoing the Fontan operation have primarily shown sinusoidal dilatation, distorted architecture and fibrosis, without evidence of significant inflammation. ${ }^{10,15,16}$ The patients who had BCPC were recruited for study in a steady state, 

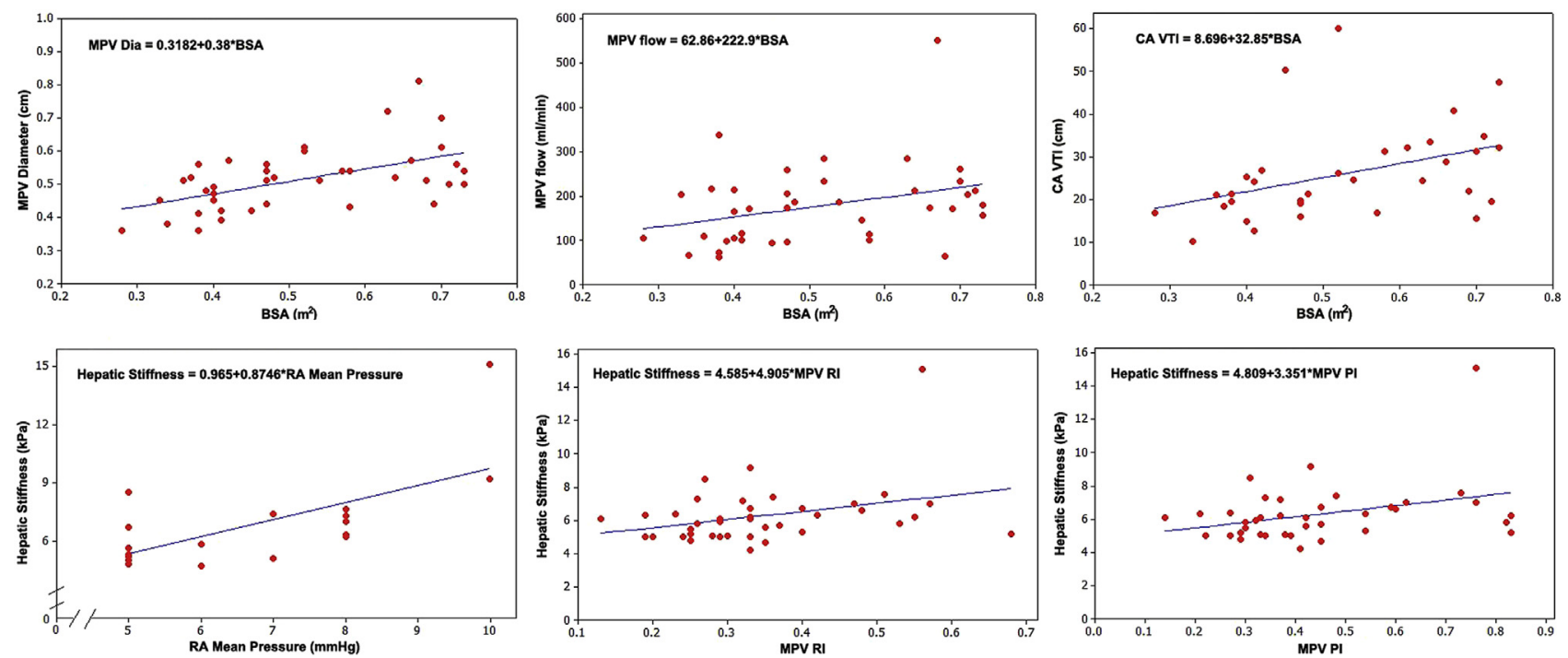

FIGURE 2. Top row shows correlations of MPV diameter, MPV flow, and CA VTI with BSA in patients and healthy subjects. Bottom row shows correlations of hepatic stiffness with MPV resistance and PIs, and with RA pressure in patients. MPV, Main portal vein; Dia, diameter; BSA, body surface area; $C A$, celiac artery; $V T I$, velocity-time integral; $R A$, right atrial; $R I$, resistive index; $P I$, pulsatility index.

as outpatients after their surgery for BCPC, so the influence of inflammation or acute illness on hepatic stiffness is expected to have been minimized. Inter- and intraobserver variability in stiffness measurements could be important clinically, so standalone stiffness determinations should be interpreted with caution. Replicated averaged determinations may provide more clinical value than a single measurement.

\section{CONCLUSIONS}

Hepatic stiffness is increased with BCPC physiology, and this finding raises concerns that hepatopathology in palliated single-ventricle CHD is not attributable to exclusively Fontan physiology. Hepatic stiffness measurements using SWE are feasible in this young population, and the technique shows promise as a means for monitoring disease progression. Hepatic stiffness measurements may find application in other forms of congenital heart disease, in which the right heart is abnormally burdened, including ventricular inversion with levo-transposition of the great arteries, surgically modified tetralogy of Fallot, and Ebstein's anomaly. Longitudinal assessments of hepatic stiffness in this cohort with single-ventricle CHD, in conjunction with serum fibrosis markers (enhanced liver fibrosis and those found with FibroSure; LabCorp, Burlington, NC) and a search for gene mutations that may predispose patients to liver disease (HFE and alpha 1-antitrypsin), are ongoing.

\section{Conflict of Interest Statement}

Authors have nothing to disclose with regard to commercial support.
The authors are grateful to the patients who participated in this study. The authors appreciate the assistance of Carolyn Chamberlain, RN, MPH; Sara Warta, RN, BSN, Nicole Hardin, MS, RT; Rallyn Renner, RDMS; Kris Houston, RN; and Ling Li, MD, PhD.

\section{References}

1. Wu FM, Ukomadu C, Odze RD, Valente AM, Mayer JE Jr, Earing MG. Liver disease in the patient with Fontan circulation. Congenit Heart Dis. 2011;6:190-201.

2. Rychik J, Veldtman G, Rand E, Russo P, Rome JJ, Krok K, et al. The precarious state of the liver after a Fontan operation: summary of a multidisciplinary symposium. Pediatr Cardiol. 2012;33:1001-12.

3. Asrani SK, Asrani NS, Freese DK, Phillips SD, Warnes CA, Heimbach J, et al. Congenital heart disease and the liver. Hepatology. 2012;56:1160-9.

4. Camposilvan S, Milanesi O, Stellin G, Pettenazzo A, Zancan L, D’Antiga L. Liver and cardiac function in the long term after Fontan operation. Ann Thorac Surg. 2008;86:177-82.

5. Gewillig M, Goldberg DJ. Failure of the Fontan circulation. Heart Fail Clin 2014;10:105-16.

6. Krieger EV, Moko LE, Wu F, Landzberg MJ, Valente AM, Assenza GE, et al. Single ventricle anatomy is associated with increased frequency of nonalcoholic cirrhosis. Int J Cardiol. 2013;167:1918-23.

7. Ghaferi AA, Hutchins GM. Progression of liver pathology in patients undergoing the Fontan procedure: chronic passive congestion, cardiac cirrhosis, hepatic adenoma, and hepatocellular carcinoma. J Thorac Cardiovasc Surg. 2005;129: $1348-52$.

8. Asrani SK, Warnes CA, Kamath PS. Hepatocellular carcinoma after the Fontan procedure. N Engl J Med. 2013;368:1756-7.

9. Guha IN, Bokhandi S, Ahmad Z, Sheron N, Cope R, Marshall C, et al. Structural and functional uncoupling of liver performance in the Fontan circulation. Int J Cardiol. 2013;164:77-81.

10. Kutty SS, Peng Q, Danford DA, Fletcher SE, Perry D, Talmon GA, et al; Liver Adult-Pediatric-Congenital-Heart-Disease Dysfunction Study (LADS) Group. Increased hepatic stiffness as a consequence of high hepatic afterload in the Fontan circulation: a vascular Doppler and elastography study. Hepatology. 2014;59:251-60.

11. Wu FM, Opotowsky AR, Raza R, Harney S, Ukomadu C, Landzberg MJ, et al Transient elastography may identify Fontan patients with unfavorable hemodynamics and advanced hepatic fibrosis. Congenit Heart Dis. 2014;9:438-47.

12. Yoo BW, Choi JY, Eun LY, Park HK, Park YH, Kim SU. Congestive hepatopathy after Fontan operation and related factors assessed by transient elastography. $J$ Thorac Cardiovasc Surg. 2014;148:1498-505. 
13. Kiesewetter CH, Sheron N, Vettukattill JJ, Hacking N, Stedman B, MillwardSadler H, et al. Hepatic changes in the failing Fontan circulation. Heart. 2007; 93:579-84

14. Schwartz MC, Sullivan L, Cohen MS, Russo P, John AS, Guo R, et al. Hepatic pathology may develop before the Fontan operation in children with functional single ventricle: an autopsy study. J Thorac Cardiovasc Surg. 2012;143:904-9.

15. Kendall TJ, Stedman B, Hacking N, Haw M, Vettukattill JJ, Salmon AP, et al. Hepatic fibrosis and cirrhosis in the Fontan circulation: A detailed morphological study. J Clin Pathol. 2008:61:504-8.
16. Schwartz MC, Sullivan LM, Glatz AC, Rand E, Russo P, Goldberg DJ, et al Portal and sinusoidal fibrosis are common on liver biopsy after Fontan surgery. Pediatr Cardiol. 2013;34:135-42.

Key Words: congenital heart disease, Fontan procedure, bidirectional cavopulmonary anastomosis, hepatic stiffness, shear wave elastography

Readers who found these articles interesting may also like to read the following papers found in recent and future issues of our sister publications, Seminars in Thoracic and Cardiovascular Surgery and Operative Techniques in Thoracic and Cardiovascular Surgery!

\section{Congenital}

State of the Art: Interrupted Aortic Arch. Richard Jonas. Semin Thorac Cardiovasc Surg 2015; Summer; 27(2):177-188.

Original Submission: Should Tricuspid annuloplasty be performed with pulmonary valve replacement for pulmonary regurgitation in repaired tetralogy of fallot? Pranava Sinha. Semin Thorac Cardiovasc Surg 2015; Summer; 27(2): 159-165.

Editorial Commentary: PI Leads to RVE, Which Leads to TR, Which Leads to More RVE, Which Leads to More TR: A Vicious Cycle? Charles B. Huddleston. Semin Thorac Cardiovasc Surg 2015; Summer; 27(2): 166-167.

Original Submission: Unsatisfactory early and late outcomes after Fontan surgery delayed to adolescence and adulthood. Yves D’Udekem. Semin Thorac Cardiovasc Surg 2015; Summer; 27(2):168-174.

Editorial Commentary: The Fontan Operation: Is Timing Everything? Harold M Burkhart. Semin Thorac Cardiovasc Surg 2015; Summer; 27(2): 175.

Discussions in Cardiothoracic Treatment and Care: Tetralogy of Fallot. Charles Fraser. Semin Thorac Cardiovasc Surg 2015; Summer; 27(2):189-204.

Current Readings: Pulmonary Valve Replacement for Pulmonary Valve Insufficiency in Formerly Repaired Tetralogy of Fallot. Brian Kogon. Semin Thorac Cardiovasc Surg 2015; Spring; 27(1):57-64.

Original Submission: Preliminary experience in the use of an extracellular matrix (CorMatrix) as a tube graft: Word of caution. Narutoshi Hibino. Expected Publication December 2015.

Original Submission: Relationship of normal aortic valve cusp dimensions: a tool to optimize cusp reconstruction valvuloplasty. Sujata Subramanian. Expected Publication December 2015.

Original Submission: Outcomes of truncus arteriosus repair in children: 35 years of experience from a single institution. Igor Konstantinov. Expected Publication December 2015.

Original Submission: Efficacy of artificial chordal reconstruction for idiopathic severe mittral regurgitation due to chordal rupture in infancy. Sadahiro Sai. Expected Publication December 2015.

Original Submission: The mid-term outcomes of bioprosthetic pulmonary valve replacement in children. Takeshi Shinkawa. Expected Publication December 2015.

Original Submission: Health-related quality of life in adult survivors after the Fontan operation. James Kirklin. Expected Publication December 2015.

Repair of Atrioventricular Septal Defects: The 2-Patch Sandwich Technique. Hagi Dekel. Oper Tech Thorac Cardiovasc Surg 2015; Spring; 20(1):63-74.

Classic Single-Patch Repair of Atrioventricular Septal Defects. Anees J. Razzouk. Oper Tech Thorac Cardiovasc Surg 2015; Spring; 20(1):75-86.

Melody Valve for Mitral Valve Replacement. Sitaram M. Emani. Oper Tech Thorac Cardiovasc Surg 2014; Winter; 19(4):454-463. Single-Stage Neonatal Repair of Taussig-Bing Anomaly. Bahaaldin Alsoufi. Oper Tech Thorac Cardiovasc 2014; Winter; 19(4): 464-481

Repair of Anomalous Coronary Artery from the Pulmonary Artery by Aortic Implantation. Anthony Azakie. Expected Publication December 2015 